
\title{
$\begin{array}{ll}\text { Research Square } & \text { Preprints are preliminary reports that have not undergone peer review. } \\ \text { They should not be considered conclusive, used to inform clinical practice, } \\ \text { or referenced by the media as validated information. }\end{array}$
}

\section{Risk factors for bladder calculus in patients with benign prostatic hyperplasia: a retrospective single-center study}

\section{Xin Sun}

The Second Hospital of Anhui Medical University

Dexin Yu ( $\square$ yudx_urology@126.com)

Department of Urology, The Second Hospital of Anhui Medical University, Hefei, China

Zhangjun Cao

The Second Hospital of Anhui Medical University

\section{Dongdong Xie}

The Second Hospital of Anhui Medical University

\section{Liangkuan $\mathrm{Bi}$}

The Second Hospital of Anhui Medical University

\section{Research article}

Keywords: Bladder calculus · Benign prostatic hyperplasia $\cdot$ Risk factor · Intravesical prostatic protrusion · Acute urinary retention · Serum creatinine

Posted Date: February 26th, 2020

DOI: https://doi.org/10.21203/rs.2.24560/v1

License: @ (1) This work is licensed under a Creative Commons Attribution 4.0 International License. Read Full License 


\begin{abstract}
Background: To explore the risk factors that lead to the formation of bladder calculus in patients with benign prostatic hyperplasia (BPH). Methods: Retrospective study was performed between June 2017 and October 2019, 103 patients with BPH who underwent transurethral resection of the prostate (TURP) were included. Patients with BPH were divided into two groups: 32 patients with bladder calculus in group1 and 71 patients without bladder calculus in group2. Characteristics of both groups were compared and univariate and multivariate analyses were performed to investigate the association between BPH with or without bladder calculus. Results: There was no significant difference between the two groups regarding age, duration of $\mathrm{BPH}$, body mass index (BMI), total prostate volume (TPV), total prostate-specific antigen (TPSA), International prostate symptom score (IPSS), serum albumin, hemoglobin, uric acid, urinary tract infection, diabetes, and hypertension. The patients' serum creatinine and acute urinary retention (AUR) were significantly lower, while intravesical prostatic protrusion (IPP) was significantly higher in group 1 than group 2 . Multivariate analyses revealed that IPP and AUR were significant risk factors for the formation of bladder calculus in patients with BPH. For IPP, the receiver operating characteristic (ROC) curve showed that the cut-off value of the highest risk of the formation of bladder calculus was 11.5 $\mathrm{mm}$. Conclusions: Our study indicated that IPP and AUR were independent risk factors that were closely linked with the incidence of bladder calculus in BPH patients.
\end{abstract}

\title{
Background
}

As a common complication of $\mathrm{BPH}$, bladder calculus comprises $5 \%$ of urinary calculus diseases [1]. With the improvement of nutritional supplements and the widespread use of modern antibiotics, the incidence of bladder calculus has decreased significantly in recent decades [2]. BPH is highly prevalent in elderly men aging from 60 to 70 years old [3]. BPH accompanied by bladder calculus was an absolute surgical indication [4]. Bladder outlet obstruction (BOO) has been considered as a predisposing factor for the formation of bladder calculus in patients $[5,6]$. It has been reported that bladder calculus accounts for $3 \%-8 \%$ of patients with BOO as a result of $\mathrm{BPH}$ [6-8]. Though few studies have indicated that IPP, age, Qmax, and urinary tract infection may influence the formation of bladder calculus in BPH patients, little knowledge is known about the etiology between these two diseases $[9,10]$. Therefore, this study aimed to analyze the risk factors that facilitate the formation of bladder calculus in BPH patients.

\section{Patients And Methods Study Population}

This retrospective study was conducted at the Second Hospital of Anhui Medical University, Department of Urology between June 2017 and October 2019. This study design was approved by the Institutional Review Board of the Second Hospital of Anhui Medical University and is adhered to the Declaration of Helsinki. A total of 103 consecutive male patients with BPH underwent TURP were screened and were divided into two groups: group 1 (BPH with bladder calculus) and group 2 (BPH without bladder calculus). Patients were enrolled when they had unsuccessful medical histories with the treatment of alpha-blockers or 5-alpha reductase inhibitors and finally received TURP. Those patients confirmed prostate malignant tumor, bladder cancer, bladder foreign body, urethral stricture, bilateral hydronephrosis, neurogenic bladder, previous history of urolithiasis, and patients with missing data were excluded from the study.

\section{Measurements And Outcomes}

Perioperative parameters of age, BMI, duration of BPH, IPSS, TPSA, TPV, IPP, serum creatinine, serum albumin, hemoglobin, uric acid, AUR, urinary tract infection, diabetes, and hypertension. IPP was measured by sonography and defined as the distance between the tip of the prostate median lobe and the bladder neck in the midsagittal plane [11]. All patients underwent TURP with or without endoscopic lithotripsy.

\section{Statistical Analysis}

The results were recorded in EXCEL spreadsheets (Microsoft, Seattle, WA USA) and analyzed using the Statistical Package for the Social Sciences (SPSS) version 22.0 software (IBM Corp, Armonk, NY, USA). Continuous data were analyzed using mean values with standard deviations and compared by Student's t-tests. Categorical data were analyzed by the Pearson chi-square tests. To assess 
whether AUR, serum creatinine, or IPP was an independent risk factor for BPH patients with bladder calculus, logistic regression analyzes and ROC curve was utilized. Differences were considered significant when $\mathrm{P}<0.05$.

\section{Results}

Of the 103 patients, group 1 included 32 patients and group 2 included 71 patients. The overall rate of bladder calculus in BPH patients was $31.1 \%$. The maximum diameter of bladder calculus was $22 \pm 2 \mathrm{~mm}$, and half of the patients have more than two calculus in group $1(n=16)$. As shown in Table 1 , there were no significant changes in age, duration of BPH, BMI, TPV, TPSA, IPSS, serum albumin, hemoglobin, uric acid, urinary tract infection, diabetes, and hypertension in either group. The patients' serum creatinine (75.8 vs $88.0, p=0.043)$ and AUR $(9.4 \%$ vs $29.6 \%, p=0.025)$ were lower, while IPP ( 12.7 vs 9.0, $p=0.002)$ were higher in group 1 than group 2.

As shown in Table 2, univariate analysis revealed that the presence of bladder calculus was associated with serum creatinine $(p=0.049)$, AUR $(p=0.045)$, and IPP $(p=0.008)$. Multivariate analysis showed that IPP $(p=0.006)$ and AUR $(p=0.044)$ were statistically significant predictors for bladder calculus in BPH patients. There was no statistically significant $(p=0.083)$ relationship between serum creatinine and bladder calculus in our study.

We then used the ROC curve to analyze the correlation of IPP or AUR with bladder calculus. ROC curve showed that IPP was a more important risk factor compared with AUR. The area under the curve (AUC) of IPP was 0.699, larger than AUC of AUR (0.399). The cutoff point of IPP was determined as $11.5 \mathrm{~mm}$. The sensitivity and specificity were $70 \%$ and $59.4 \%$, respectively (Fig. 1).

\section{Discussion}

Bladder calculus, as one of the most common complications of BPH, the incidence is approximately $10 \%$ [1]. BOO, urinary tract infection, neurogenic bladder, bladder diverticula, bladder augmentation, or the bladder foreign body have been shown may be associated with the occurrence of bladder calculus [12]. Combining bladder stones is also an absolute surgical indication of BPH. In undeveloped areas, many patients with BPH hesitate to receive surgical treatment, insisting on drug conservative treatment even when bladder calculus has formed, which causes great harm to physical health and severely affects the quality of life during disease progression. Researchers have been troubled by the problem of why some BPH patients have a higher possibility accompany bladder calculus for many years. Though few studies have explored some risk factors of bladder calculus formation in patients with $\mathrm{BPH}$, which indicated IPP seems to be the most important potential risk factor, the result is still uncertain $[9,10]$.

In this study, we aimed to determine the risk factor that influences the presence of bladder calculus in patients with BPH.

The concept of IPP was proposed by Jean Casimir Félix Guyon in 1888 in Paris [13]. After two centuries of research, IPP has emerged as a novel prostatic morpho-metric parameter of significance to help the clinicians in many aspects of managing the patients with some diseases of the lower urinary tract and the prostate [14]. Topazio et al found that IPP was significantly and inversely correlated with treatment success in patients with lower urinary tract symptoms (LUTS) and BPH under alpha-blockers therapy. IPP can be used as a predictor of alpha-blockers response [15]. A recent study indicated that IPP was a good predictor of clinically significant BOO due to BPH [16]. Lee also showed that even in men with good flow, high grades of IPP were more likely to have B0O [17]. Lim et al reported that a long IPP would cause BOO by creating a "ball-valve" effect during voiding, which destroy the funneling effect of the bladder neck [18]. Chia et al also demonstrated that IPP was caused by the enlarging lateral lobes and the median lobe, and that the protrusion of the enlarged lobes caused a "ball-valve" type of obstruction, thus disrupting the funneling effect of the bladder neck and causing dyskinetic movement of the bladder during voiding [19]. In this study, the mean length of the IPP was $12.7 \mathrm{~mm}$ in group 1 and $9 \mathrm{~mm}$ in group 2. The statistically significant association between these two groups shows that IPP was a risk factor for bladder calculus formation in BPH patients. In a prospective clinical study, Mariappan et al indicated that IPP can be divided into three levels based on the length of the IPP [20]. Grade I, the length of IPP was less than $5 \mathrm{~mm}$; Grade II, the length of IPP was 5 to $10 \mathrm{~mm}$; and Grade III, the length of IPP was longer than $10 \mathrm{~mm}$. In our study, all patients in group 1 and 2 were classified into Grade II and III.

ROC curve revealed that the cut-off value of IPP that caused the highest risk of bladder calculus formation was $11.5 \mathrm{~mm}$. Kim et al reviewed data of $271 \mathrm{BPH}$ patients, the median length of IPP was $11.5 \mathrm{~mm}$ in patients combing with bladder stones, while the average length was $3.4 \mathrm{~mm}$ in patients without bladder stones [9]. In another study, the median length of IPP was $11 \mathrm{~mm}$ in BPH patients with bladder calculus, while $4 \mathrm{~mm}$ in patients without calculus [10]. Lu et al indicated that, for IPP, the cut-off value of the highest risk of hydronephrosis was $1.95 \mathrm{~cm}$. Interestingly, the median length of IPP in their study receiving TURP was longer than ours [11]. In our 
study, the length of all IPP was $\geq 5 \mathrm{~mm}$, and all patients received surgical intervention. These results make the length of IPP $\geq 5 \mathrm{~mm}$ the potential to become a novel indication for TURP surgery. Inconsistent with previous studies, univariate, multivariate analyses, and the ROC curve indicated that IPP was an important independent risk factor for the presence of bladder calculus in BPH patients $[9,10]$.

AUR is a urological emergency, it usually presents as a sudden inability to urinate with the symptom of lower abdominal pain [21]. The risk is cumulative and increases with age. About $10 \%$ of old men in their $70 \mathrm{~s}$ and $30 \%$ in their $80 \mathrm{~s}$ will develop AUR within 5 years [22]. AUR is significantly associated with morbidity, mortality, and cost to the public health service. On the basis of the guidelines of the European Association of Urology, initial management of AUR should consist of bladder decompression with an indwelling catheter followed by trial without catheter [23]. The pathogenesis of AUR can be multifactorial. Chen et al revealed that BPH causes the most AUR, and the incidence is estimated to be as high as $53 \%$ [24]. AUR is also associated with bladder calculus. When bladder calculus causes posterior urethral obstruction, AUR also occurs. A common manifestation in children is interrupted urination, and urination can continue after changing positions or rubbing the penis. In our study, the AUR rate was significantly higher in group 2 than in group 1.3 patients $(9.1 \%)$ in group 1 had AUR, while 21 patients (30\%) in group 2 had AUR. The results of our study showed that AUR was an independent risk factor for the occurrence of bladder calculus in patients with BPH. Further ROC curve demonstrated that AUR was the second most important risk factor compared with IPP. A similar AUR rate was also found in a previous study. Huang et al reported that the AUR rate was $10.6 \%$ in patients with $\mathrm{BPH}$ and bladder calculus, lower than $37.4 \%$ in patients with BPH. However, AUR was not an independent risk factor in their study [10]. Confusingly, in spite of bladder calculus or BPH can cause AUR, the AUR rate in group 1 was lower. We hypothesized that it may be attributed to patients with BPH developed AUR early and received standardized medications, the probability of complications with bladder stones was greatly reduced. From our perspectives, severe BPH symptoms and no history of AUR may be an important sign of combining with bladder calculus. Due to the limited number of patients in our study, further studies are still warranted to be explored this problem.

Now few studies have focused on the relationship between serum creatinine and bladder calculus. Univariate analyses showed serum creatinine was a potential risk factor, however, multivariate analyses revealed that it was not an independent risk factor in comparison with IPP and AUR. A lower level of serum creatinine was found in group 1. Li et al indicated that lower levels of serum creatinine and uric acid, and lower percentages of diabetes mellitus were local factors in the formation of bladder uric acid stone [25]. Interestingly, the level of uric acid was also lower in group 1, though results showed it was not an independent risk factor. Older age and higher rates of urinary tract infection have also been considered as independent risk factors in previous studies $[9,10]$. In our study, patients in group 1 were a little younger and the rate of urinary tract infection was a little higher than patients in group 2. However, age and urinary tract infection were proven not to be independent risk factors. Moreover, our results also first demonstrated that serum albumin, hemoglobin, diabetes, hypertension, smoking, and drinking were not independent risk factors for the occurrence of bladder calculus in patients with $\mathrm{BPH}$.

The limitations of this study should be noted. First, it has a retrospective design with a relatively small sample size and its inherent retrospective and non-randomized nature may have led to selection bias. Second, all patients enrolled in our study were Chinese, so we cannot eliminate the influence of ethnic diversity. Third, all patients did not have accurate uroflow parameters and urodynamic results, making the study hard to further exploring whether urodynamic indicators are risk factors. A prospective randomized controlled trial is urgently needed in the future.

\section{Conclusion}

IPP and AUC are independent risk factors that linked with the occurrence of bladder calculus in patients with BPH. According to the ROC curve result, in comparison with AUC, IPP was the most important risk factor. Moreover, the cut-off value of the highest risk of the formation of bladder calculus was $11.5 \mathrm{~mm}$. These indicators can help the surgeons in filtering BPH patients combine with bladder calculus.

\section{Abbreviations}

BPH: Benign prostatic hyperplasia; TURP: Transurethral resection of the prostate; BMI: Body mass index; TPV: Total prostate volume; TPSA: Total prostate-specific antigen; IPSS: International prostate symptom score; AUR: Acute urinary retention; IPP: Intravesical prostatic protrusion; ROC: Receiver operating characteristic; BOO: Bladder outlet obstruction; AUC: Area under the curve; LUTS: Lower urinary tract symptoms 


\section{Declarations}

\section{Acknowledgements}

Not applicable.

\section{Author contributions}

DY performed the development of study design, and the original literature search, and participated in drafting the manuscript. XS participated in the design of the study and performed the statistical analysis. ZC, DX, and LB participated in data acquisition and analysis. All authors read and approved the final manuscript.

\section{Funding}

No funding was obtained for this study.

\section{Availability of data and materials}

The datasets used and/or analyzed during the current study are available from the corresponding author on reasonable request.

\section{Ethics approval and consent to participate}

This research is retrospective and involving the collection of existing data and records. The ethical committee of the Second Hospital of Anhui Medical University approved all procedures of the study and written informed consent was obtained from all cases.

\section{Consent for publication}

Consent was obtained.

\section{Competing interests}

The authors declare that they have no competing interests.

\section{Author details}

Department of Urology, the Second Hospital of Anhui Medical University, 80 Feicui Rd, Hefei, Anhui 230032, PR China

\section{References}

1. Papatsoris AG, Varkarakis I, Dellis A, Deliveliotis C. Bladder lithiasis: from open surgery to lithotripsy. Urol Res. 2006;34(3):163-7.

2. Philippou P, Ralph DJ, Timoney AG. The impact of shock wave lithotripsy on male fertility: a critical analysis of existing evidence. Urology. 2012;79(3):492-500.

3. Shah HN, Hegde SS, Shah JN, Mahajan AP, Bansal MB. Simultaneous transurethral cystolithotripsy with holmium laser enucleation of the prostate: a prospective feasibility study and review of literature. BJU Int. 2007;99(3):595-600.

4. Gratzke C,Bachmann A, Descazeaud A, Drake MJ, Madersbacher S, Mamoulakis C, et al. EAU Guidelines on the Assessment of Non-neurogenic Male Lower Urinary Tract Symptoms including Benign Prostatic Obstruction. Eur Urol. 2015;67(6):1099-109.

5. Kuo TL, Teo JS, Foo KT. The role of intravesical prostatic protrusion (IPP) in the evaluation and treatment of bladder outlet obstruction (BOO). Neurourol Urodyn. 2016; 35(4):535-7.

6. Krambeck AE, Rule AD, Li X, Bergstralh EJ, Gettman MT, Lieske JC. Shock wave lithotripsy is not predictive of hypertension among community stone formers at long-term follow up. J Urol. 2011;185(1):164-9.

7. Li XD, Wu YP, Ke ZB, Lin TT, Chen SH, Xue XY, et al. Predictors Of Postoperative Lower Urinary Tract Symptoms Improvements In Patient With Small-Volume Prostate And Bladder Outlet Obstruction. Ther Clin Risk Manag. 2019;15:1291-304.

8. Childs MA, Mynderse LA, Rangel LJ, Wilson TM, Lingeman JE, Krambeck AE. Pathogenesis of bladder calculi in the presence of urinary stasis. J Urol. 2013;189(4):1347-51. 
9. Kim JW, Oh MM, Park HS, Cheon J, Lee JG, Kim JJ, et al. Intravesical prostatic protrusion is a risk factor for bladder stone in patients with benign prostatic hyperplasia. Urology. 2014;84(5):1026-9.

10. Huang W, Cao JJ, Cao M, Wu HS, Yang YY, Xu ZM, et al. Risk factors for bladder calculi in patients with benign prostatic hyperplasia. Medicine (Baltimore). 2017;96(32):e7728.

11. Lu CH, Wu HHH, Lin TP, Huang YH, Chung HJ, Kuo JY, et al. Is intravesical prostatic protrusion a risk factor for hydronephrosis and renalinsufficiency in benign prostate hyperplasia patients? J Chin Med Assoc. 2019;82(5):381-4.

12. Yoshida O, Okada Y. Epidemiology of urolithiasis in Japan: a chronological and geographical study. Urol Int. 1990;45:104-11.

13. Guyon JCF. Clinical lectures on surgical disorders of bladder and prostate. Paris:J.B.Baillie're. 1888.

14. Mehraban D. Clinical value of intravesical prostatic protrusion in the evaluation and management of prostatic and other lower urinary tract diseases. Asian J Urol. 2017;4(3):174-80.

15. Topazio L, Perugia C, De Nunzio C, Gaziev G, lacovelli V, Bianchi D, et al. Intravescical prostatic protrusion is a predictor of alpha blockers response: results from an observational study. BMC Urol. 2018;18(1):6.

16. Reddy SVK, Shaik AB. Non-invasive evaluation of bladder outlet obstruction in benign prostatic hyperplasia: a clinical correlation study. Arab J Urol. 2019;17(4):259-64.

17. Lee A, Lee HJ, Lim KB, Huang HH, Ho H, Foo KT. Can intravesical prostatic protrusion predict bladder outlet obstruction even in men with good flow? Asian J Urol. 2016;3(1):39-43.

18. Lim KB, Ho H, Foo KT, Wong MY, Fook-Chong S. Comparison of intravesical prostatic protrusion, prostate volume and serum prostatic-specific antigen in the evaluation of bladder outlet obstruction. Int J Urol. 2006;13(12):1509-13.

19. Chia SJ, Heng CT, Chan SP, Foo KT. Correlation of intravesical prostatic protrusion with bladder outlet obstruction. BJU Int. 2003;91:371-4.

20. Mariappan P, Brown DJ, McNeill AS. Intravesical prostatic protrusion is better than prostate volume in predicting the outcome of trial without catheter in white men presenting with acute urinary retention: a prospective clinical study. J Urol. 2007;178:573-7.

21. Izard J, Nickel JC. Impact of medical therapy on transurethral resection of the prostate: two decades of change. BJU Int. 2011;108(1):89-93.

22. Jacobsen SJ, Jacobson DJ, Girman CJ, Roberts RO, Rhodes T, Guess HA, et al. Natural history of prostatism: risk factors for acute urinary retention. J Urol. 1997;158(2):481-7.

23. Patel PM, Sweigert SE, Nelson M, Gupta G, Baker M, Weaver FM, et al. Disparities in BPH Progression: Predictors of Presentation to the Emergency Department in Urinary Retention. J Urol. 2020;101097JU0000000000000787.

24. Chen JS, Chang $\mathrm{CH}$, Yang WH, Kao YH. Acute urinary retention increases the risk of complications after transurethral resection of the prostate: a population-based study. BJU Int. 2012;110:E896-901.

25. Li WM, Chou YH, Li CC, Liu CC, Huang SP, Wu WJ, et Local factors compared with systemic factors in the formation of bladder uric acid stones. Urol Int. 2009;82(1):48-52.

\section{Tables}

Table 1 Comparison of baseline characteristics between group 1 and group 2 


\begin{tabular}{llll}
\hline Perioperative parameters & Group 1 $(\mathrm{N}=32)$ & Group 2 $(\mathrm{N}=71)$ & P value \\
\hline Age (year) & $71.25 \pm 1.39$ & $71.55 \pm 0.80$ & 0.585 \\
\hline BMI $(\mathrm{kg} / \mathrm{m} 2)$ & $23.38 \pm 0.62$ & $23.61 \pm 0.39$ & 0.753 \\
\hline Duration of BPH (month) & $23.50 \pm 5.18$ & $21.51 \pm 2.92$ & 0.722 \\
\hline IPSS & $22.28 \pm 0.42$ & $21.55 \pm 0.38$ & 0.732 \\
\hline PSA $(\mathrm{ng} / \mathrm{ml})$ & $2.80 \pm 0.16$ & $2.90 \pm 0.18$ & 0.669 \\
\hline TPV $(\mathrm{g})$ & $45.80 \pm 1.89$ & $49.52 \pm 1.78$ & 0.157 \\
\hline IPP $(\mathrm{mm})$ & $12.71 \pm 0.09$ & $8.99 \pm 0.06$ & 0.002 \\
\hline Serum albumin $(\mathrm{g} / \mathrm{L})$ & $36.53 \pm 0.56$ & $36.99 \pm 0.57$ & 0.567 \\
\hline Hemoglobin $(\mathrm{g} / \mathrm{L})$ & $135.84 \pm 2.55$ & $131.99 \pm 1.76$ & 0.221 \\
\hline Serum creatinine $(\mu \mathrm{mol} / \mathrm{L})$ & $75.75 \pm 2.97$ & $88.04 \pm 3.79$ & 0.043 \\
\hline Uric acid $(\mu \mathrm{mol} / \mathrm{L})$ & $313.56 \pm 14.11$ & $328.35 \pm 8.84$ & 0.364 \\
\hline Urinary tract infection, $\mathrm{n}(\%)$ & $15 \square 46.9 \% \square$ & $31 \square 43.7 \% \square$ & 0.761 \\
\hline AUR, $\mathrm{n}(\%)$ & $3 \square 9.4 \% \square$ & $21 \square 21.6 \% \square$ & 0.025 \\
\hline Diabetes, $\mathrm{n}(\%)$ & $4 \square 12.5 \% \square$ & $11 \square 15.5 \% \square$ & 0.69 \\
\hline Hypertension, n $(\%)$ & $15 \square 46.9 \% \square$ & $36 \square 50.7 \% \square$ & 0.719
\end{tabular}

AUR Acute urinary retention, $B M I$ Body mass index, $B P H$ Benign prostatic hyperplasia, IPP Intravesical prostatic protrusion, IPSS International Prostate Symptom Score, PSA Prostate specific antigen, TPVTotal prostate volume

Table 2 Univariate and multivariate analysis of patients' data between group 1 and group 2

\begin{tabular}{|c|c|c|c|c|c|}
\hline Perioperative parameters & B & SE & $P$ value & $\operatorname{Exp}(\mathrm{B})$ & $95 \% \mathrm{CI}$ \\
\hline \multicolumn{6}{|l|}{ Univariate } \\
\hline Age (year) & 0.021 & 0.039 & 0.585 & 1.201 & $0.947-1.102$ \\
\hline BMI $(\mathrm{kg} / \mathrm{m} 2)$ & -0.022 & 0.078 & 0.775 & 0.978 & $0.84-1.139$ \\
\hline IPSS & -0.03 & 0.096 & 0.752 & 0.97 & $0.803-1.171$ \\
\hline PSA $(\mathrm{ng} / \mathrm{ml})$ & 0.305 & 0.203 & 0.133 & 1.357 & $0.911-2.019$ \\
\hline $\mathrm{IPP}(\mathrm{mm})$ & -1.281 & 0.479 & 0.008 & 0.278 & $0.109-0.711$ \\
\hline Serum albumin $(g / L)$ & 0.068 & 0.067 & 0.312 & 1.07 & $0.939-1.22$ \\
\hline Serum creatinine $(\mu \mathrm{mol} / \mathrm{L})$ & 0.025 & 0.013 & 0.049 & 1.026 & $1-1.052$ \\
\hline Urinary tract infection, $\mathrm{n}(\%)$ & -0.514 & 0.552 & 0.352 & 0.598 & $0.203-1.765$ \\
\hline AUR, n (\%) & 1.414 & 0.758 & 0.045 & 1.612 & $1.036-2.301$ \\
\hline \multicolumn{6}{|l|}{ Multivariate } \\
\hline IPP (mm) & -1.128 & 0.41 & 0.006 & 0.324 & $0.145-0.723$ \\
\hline Serum creatinine $(\mu \mathrm{mol} / \mathrm{L})$ & 0.023 & 0.013 & 0.083 & 1.023 & $0.997-1.049$ \\
\hline AUR, n (\%) & 1.388 & 0.693 & 0.044 & 1.534 & $1.011-2.251$ \\
\hline
\end{tabular}

$A U R$ Acute urinary retention, $B$ Regression coefficient, $B M I$ Body mass index, $C I$ Confidence interval, Exp (B) Odds ratio, IPP Intravesical prostatic protrusion, IPSS International Prostate Symptom Score, PSA Prostate specific antigen, SE Standard error.

\section{Figures}




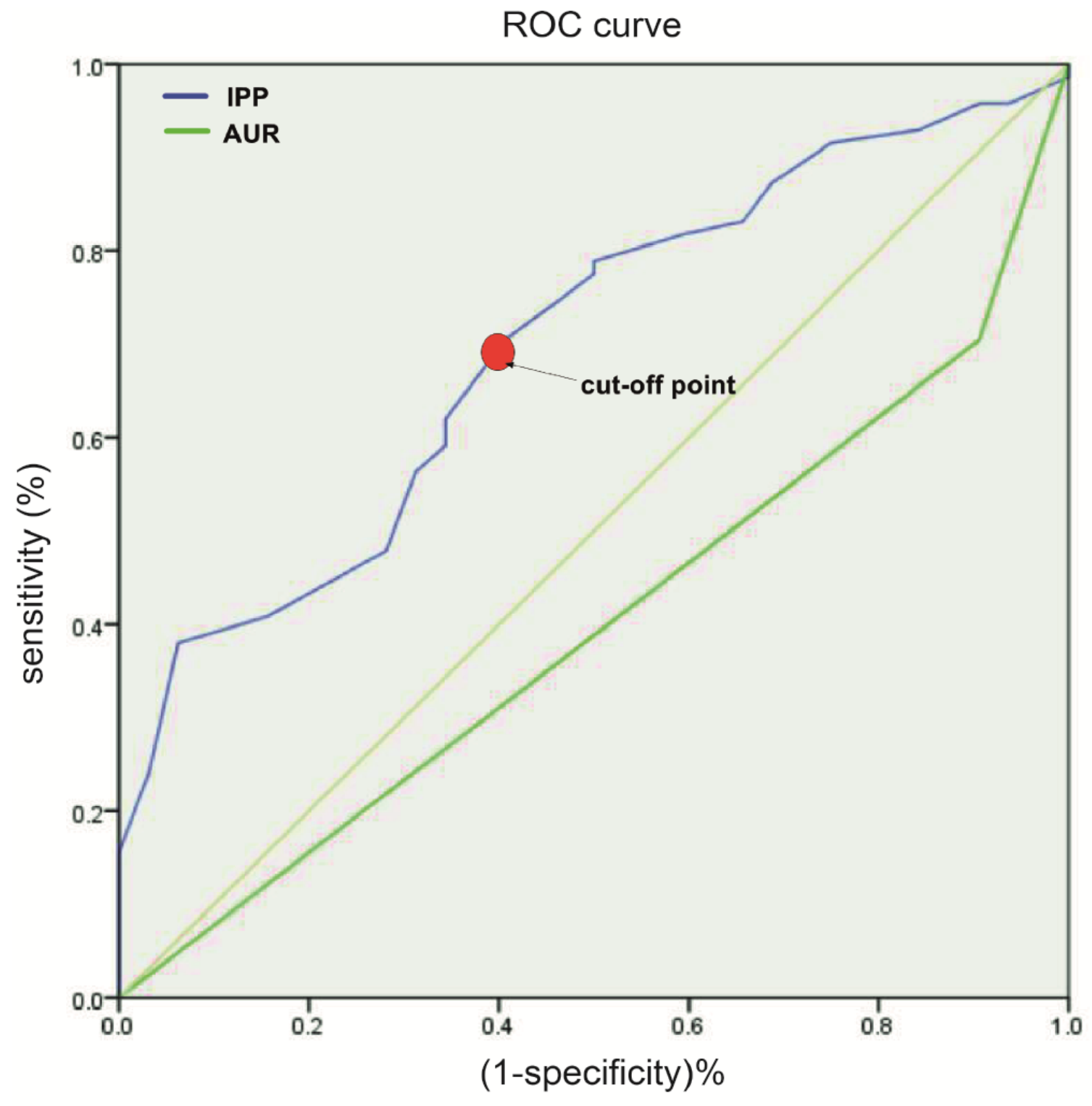

cut-off point: sensitivity $=70 \%$, specificity $=59.4 \%$

Figure 1

ROC curve and cut-off point: the association between IPP, AUR and bladder calculus in patients with BPH. 